\title{
A Conversation Between Susanne Schaffert and Ken Herrmann
}

\author{
Susanne Schaffert ${ }^{1}$ and Ken Herrmann ${ }^{2}$ \\ ${ }^{I}$ Novartis International AG, Basel, Switzerland; and ${ }^{2}$ Universitatsklinikum Essen, Essen, Germany
}

$\mathbf{J}$ ohannes Czernin, MD, editor in chief of The Journal of $\mathrm{Nu}$ clear Medicine, recently initiated a series of recorded discussions with leaders in nuclear medicine and molecular imaging. This month he asked Ken Herrmann, MD, MBA, a professor of nuclear medicine at the Universitätsklinikum Essen (Germany) to talk with Susanne Schaffert, $\mathrm{PhD}$, who became the CEO of Novartis Oncology in January. Dr. Schaffert holds a doctorate in organic chemistry from the University of Erlangen (Germany). She joined Novartis more than 20 years ago and served as Region Head, Novartis Oncology Europe, from 2012 to 2018. Other leadership positions at Novartis have included Head of Investor Relations, Global Franchise Head for Immunology and Infectious Diseases, General Manager of Novartis Oncology in Northern and Central Europe, and General Manager of Novartis Oncology in Germany. She served as chair and president of Advanced Accelerator Applications (AAA) when it was acquired by Novartis in January 2018. She is a member of the Executive Committee of Novartis and the Board of Novartis AG Germany.

Dr. Herrmann: First, thank you so much for making time available for this interview. You are the new CEO of Novartis Oncology and were previously the president of AAA. Could you tell us a little bit about your background? What was your pathway into the pharmaceutical industry?

Dr. Schaffert: I am German by nationality. I'm a chemist and hold a PhD in organic chemistry. I studied at the University of Erlangen and commenced a classic academic career. I thought my research would change the world. But I wanted to have a bigger impact and decided to join industry and go into pharmaceuticals. The close ties Novartis has with medicine and biology made me join the company in 1995. I started as a field representative, which gave me insight into how hospitals work, how clinicians think, what is needed from a product, and how to identify patient needs. At some point I started my career in general management, and my first appointment was as Head of Oncology for Novartis in Germany. At that time Novartis Oncology was quite small and had the spirit of a startup. We increased sales by more than $100 \%$. I subsequently headed the oncology organization for 28 small and midsize European countries. After moving to lead the Global Franchise of Immunology and Transplantation, I made an interesting switch when I became Head of Investor Relations for Novartis. This was definitely a very interesting experience with lots of learning. Eventually, however, I wanted to go back to business. Being close to physicians and patients was most attractive, and I resumed my role as the Head of Europe for Novartis Oncology. That was my career before I joined AAA.

COPYRIGHT @ 2019 by the Society of Nuclear Medicine and Molecular Imaging.
Dr. Herrmann: If I am correct, you were also in charge of developing Sandostatin?

Dr. Schaffert: Exactly. Rather early in my career, I was the global brand director for Sandostatin. I was then the global lead for Sandostatin LAR during its launch.

Dr. Herrmann: So you did the full circle, starting with the cold sandostatin and then...

Dr. Schaffert: ...the radioligand therapy. The concept of radioligand therapy is super exciting. It brought me back to my roots of working with neuroendocrine tumors and the patient community.

Dr. Herrmann: You recently gave an interview titled "Women in Science." We need to attract young talent and especially women into our field. I wondered what your experience was and what advice you would give to young women about entering the world of science?

Dr. Schaffert: I faced a lot of hurdles while studying chemistry in Germany, with only about $10 \%$ of students in the field being women. In my early professional years, I was always one of very few senior women-and only men sat on the executive boards. In this regard I was quite a pioneer. I advise young women to build a network. I also think that having a mentor is critical-you can help in nuclear medicine by serving as mentor.

Dr. Herrmann: On a different subject, Novartis has been in the neuroendocrine tumor space for quite some time. Your own product, Sandostatin, went off patent, and the development of radioactive versions was abandoned. What made Novartis come back into this field?

Dr. Schaffert: When I was global brand director for Sandostatin in the late 1990s, peptide-receptor radionuclide therapy (PRRT) was being performed in Europe using ${ }^{90} \mathrm{Y}$. We were looking at this concept because it was and remains quite compelling. You have a ligand hitting the target, and we found the addition of a radioactive component to be unique. However, the timing was not right and there were some toxicity concerns with ${ }^{90} \mathrm{Y}$. But we continued to watch the space. We were, of course, quite intrigued that AAA managed all the hurdles and was able to bring in expertise.

Dr. Herrmann: This was the moment that Novartis saw the chance to come back to complete what you started 20 years before?

Dr. Schaffert: Exactly. The move from yttrium to lutetium resulted in improved outcomes and a favorable safety profile. 
Also, the concept that the diagnostic completely mirrors the therapeutic is amazing. We were working closely with Mallinckrodt on the octreotide scan; but to really have a pairing between the diagnostic and the therapeutic, this was a true representative of targeted or precision medicine.

Dr. Herrmann: You referred to the NETTER-1 trial results achieved by AAA. Progression-free survival and overall survival are absolutely intriguing, but only a very small number of patients achieve complete remission or even cure. What is your vision on how to cure patients? Intensified therapy? Or combination treatments?

Dr. Schaffert: Intensifying therapy is one option. At the moment our label is limited to 4 cycles. Our current investigations look at retreatment, other radionuclides, and especially combinations-we are looking at several projects with compounds that could enhance sensitivity of radioligand therapy, maybe even immunooncology. Cure is the goal but this is a step-by-step approach.

Dr. Herrmann: Novartis also owns other targeted therapies, for example, Everolimus. Combinations sound appealing, especially for more aggressive tumors.

Dr. Schaffert: Everolimus may not be ideal to combine with a radioligand therapy, and there are no data supporting it. There are some variables we need to understand about the cell mechanisms and how we interfere in that. Many mechanisms might be considered to enhance the effect of PRRT.

Dr. Herrmann: You mentioned the limit of 4 cycles, which, of course, was initially defined by the NETTERl design. However, many centers, especially but not only in Europe, have shown that
Dr. Schaffert: Good question. I think we need to work on it. We have to identify specific criteria for when we can use an antibody. New data may come out to boost our confidence in this area. However, I'm fully aligned with you that small molecules have been proven to be a working concept. If you have the ambition to cure cancer, then antibodies may give us many more opportunities.

Dr. Herrmann: Novartis/AAA already have 2 absolutely great targets in or on their way to the clinic. What are the next 3 targets that we will also see in the clinic?

Dr. Schaffert: We are starting a phase 1 study with a newgeneration bombesin antagonist in multiple solid tumors. There we are targeting GRPR, but we need to see if the uptake is sufficient. We are also developing an agent that targets integrin $\alpha v \beta 3$ and $\alpha v \beta 5$. These are two projects we inherited from AAA. Moreover, we can now also tap into the big machine of Novartis research, with a collaboration team starting to identify new targets.

Dr. Herrmann: This sounds absolutely exciting in that it means (especially for nuclear medicine) that much more is to come. Exciting data have been published for the fibroblast-activating protein inhibitor and chemokine receptor 4. Other companies are also trying to build the data and clinical evidence needed in these targets. What is your perspective on these targets?

Dr. Schaffert: We have to see the data, of course. The concept we follow is that we do the diagnostics trial first, followed by the therapeutic trial. That's the beauty of the concept of radioligand therapy. With the diagnostic trial we already get a very good view of uptake in tumor versus tissue. This is already quite a good

"Nuclear medicine has the opportunity to become an established partner in the therapy of cancer."

patients can tolerate up to 8 and even 10 cycles quite well. Do you think that the organ-limiting dose to the kidney with 23 Gy derived from external-beam radiation therapy should be revisited?

Dr. Schaffert: You are right that a lot of these data are already out, so we could even think about it. That's why we plan to collect real-world evidence data. If we have that evidence and really can show that there are better clinical outcomes, then I think it's worth discussing with the Food and Drug Administration (FDA). But if we don't have data aggregated and collected, then it's too early. FDA wants to see that the outcome is better and safety is acceptable before having such a discussion.

Dr. Herrmann: Lutathera and the recently acquired PSMA-617 are both small molecules. Overall, there's always a big discussion about potentially using antibodies for radioligand therapy. What is your vision on the future role of antibodies?

Dr. Schaffert: Peptides and targeted small molecules have many benefits. They are shorter lived and have a superior pharmacodynamic, which go quite well with the concept of radiation. Regarding antibodies, I'm still quite interested. Antibodies offer a multitude of new targets and opportunities. Toxicity might be challenging, but we are definitely looking into antibodies.

Dr. Herrmann: A little bit more provocative question. For full disclosure, I am, of course, a big believer in small molecules and peptides. So I always say that whatever works is fine for me. However, we have been talking about antibodies for 10-15 years, yet nothing is in the clinic. What gives you faith that this would be different 5 years from now? predictive indicator of the potential of some of these compounds. It is compelling to get a lot of information in early phases-very different from other therapies in which you have to wait until later phases.

Dr. Herrmann: As a big theranostics believer, this is music to my ears. We talked a little bit about the advantages of this concept. However, there are also significant disadvantages, which is the reason it took so long for Bayer and Novartis to enter the field. Logistics are challenging: you have to consider limited half-life and shelf life, as well as a complicated supply chain. From your time at AAA, how did you overcome these challenges?

Dr. Schaffert: This is why we initially looked at AAA from the outside. Supply and logistics for commercially ready-to-use products are quite complex. We realized that AAA had the skills and the knowledge to access and control of all steps of the manufacturing process. From the isotope production to shipment of the isotope to production sites, then to radiolabeling so that you have an integrated approach-this was a critical success factor for AAA. I definitely believe there is a great advantage to having a highly integrated process of logistics and supply.

Dr. Herrmann: What you just mentioned relates to lutetium. Do you think $\alpha$-particle logistics are even more complex?

Dr. Schaffert: I don't think so, I think that a highly integrated team will be able to solve this.

Dr. Herrmann: Which $\alpha$-emitter is the most promising-actinium, radium, or thorium? 
Dr. Schaffert: I think that actinium is looking quite interesting, but we still need more data. There are also issues with limited resources and supply that need to be tackled.

Dr. Herrmann: The Journal of Nuclear Medicine obviously focuses on nuclear medicine. What is your outside perspective on how nuclear medicine has to change, for example, in regard to training and education, now that theranostics have entered the field?

Dr. Schaffert: The field of neuroendocrine tumors was a good model-very complex patients and very complex histories demanding centers of excellence with multiple specialties working together: endocrinologists, oncologists, surgeons, nuclear medicine physicians, pathologists, and more. This is really how patients should be treated. For PSMA-617 and prostate cancer, the story is quite different. Oncologists and also urologists traditionally led this field. Now a new treatment may become available bringing a new specialty to the field. Nuclear medicine has the opportunity to become an established partner in the therapy of cancer, which will require some work to facilitate. Nuclear medicine physicians need to be present in tumor boards and be committed to crossspecialty work. This requires a lot of education on both sides. Oncology needs to be aware of these potential treatments, and nuclear medicine should understand that there is now the potential for therapies in which it can be really involved. Attaining this involvement is not a given. So work needs to be done.

Dr. Herrmann: Nuclear medicine was created in internal medicine. As much as we appreciate the value of radiology and hybrid imaging, we need to be trained to think like oncologists.

Dr. Schaffert: Exactly. It starts with the training of nuclear medicine physicians. Nuclear medicine should also engage with societies like the American Society of Clinical Oncology and the European Society for Medical Oncology. You should be at these meetings, and, if there are presentations about radioligand therapies, it should be the nuclear medicine physicians presenting them. If you are not part of these societies or not working together, you will not be considered.

Dr. Herrmann: In the United States, Lutathera is already frequently used and you are preparing the rollout of PSMA. Looking at Germany, with around 80 million inhabitants and 40 theranostic centers, and translating this to the United States with a population of around 320 million, I would expect the need/potential for around 160 theranostic centers. Is this a realistic number for the United States? Do you think there is a need for dedicated theranostic centers? And does this demand justify the creation of a new (sub)specialty of theranostics as part of the evolution of nuclear medicine?

Dr. Schaffert: It is obvious that these new therapies require a certain expertise and skill. Not every hospital has the resources to do theranostics. These therapies must be done in very skilled experienced centers. We have to understand the dynamics between the involved specialties and territories. Who manages the patient? Who is the main treating physician? Is it the oncologist or the urologist? There may be concerns about which specialty decides the appropriate treatment pathway for the patient. Sometimes treatment will be given by nuclear medicine, but the lead physician would still be the oncologist or the urologist, as there may be a different therapy that comes into the mix. We really need to think through how patients are managed, especially as we start getting into different disease areas.

Dr. Herrmann: Yes, I completely agree that setting up powerful theranostic centers is essential for the success of radioligand therapy. But I do think that a special subspecialty training requirement for theranostics is something we should think about.

Dr. Schaffert: For nuclear medicine, your therapeutic focus is the radioligand therapy, whereas oncologists treat various tumors. Eventually, the hope is that radioligand therapy will become available for several different tumor types, and you will need to collaborate. Potentially you will have to consider some nuclear medicine physician specialization into specific tumor types. So it is logical to think about more specialization.

Dr. Herrmann: One major challenge is the big gap between Europe and the United States. In short, the innovation comes from Europe but the commercial revenues from the United States. Usually you want to have the innovation where the revenues come from. How can we revitalize or revive nuclear medicine and especially innovation in the United States?

Dr. Schaffert: If a therapy is approved in the market, you really see a quick closure of the gap as U.S. physicians engage in this treatment. They quickly come up with new ideas in terms of combinations and advance quite nicely. One challenge in the United States is the complexity of the regulatory process and its effect on early access to new compounds. If companies provide compounds and logistics, the United States can step up much more quickly. Providing ready-to-use products, taking care of the logistics and radioligand labeling, and so on-all this can be done by the company to accelerate the process.

Dr. Herrmann: You just mentioned the regulatory hurdles and their heterogeneity. For example, in Australia and Austria you can do more or less whatever you want, and in Germany there is a specific way to do compassionate use early on. In the United States, there is "the right to try." Do you think overall that this regulatory heterogeneity is an advantage or a disadvantage for the field of theranostics? And how do you see ideally the regulatory bodies in Europe and the United States handling development of new theranostic therapies in the future?

Dr. Schaffert: For the United States, it is very important that physicians get access early. And then, with FDA support, it is critical to establish expanded access programs or compassionate use applications so that physicians can have access early. The right to try is also something we obviously support. We currently benefit from the fact that data already exist. But for us as a company it is important that this is in agreement with the FDA, so that the data created in Europe can be used in the U.S. approval processes.

Dr. Herrmann: If you had a "free wish" from the FDA, what would this be?

Dr. Schaffert: The free wish would be that the FDA continues to support this type of very new therapy across different departments. We talked to many different people to help them understand the challenges brought by new kinds of therapy. The FDA is very collaborative, but we should also find new ways to allow even earlier access. This is critical, because it will also later ensure more patient safety.

Dr. Herrmann: I completely agree. The FDA has been marvelous for development of academic diagnostic and theranostic Investigational New Drugs. I would like to delve a little deeper into industry-academia relationships. The whole PSMA story was initially academia driven by groups like those of Martin Pomper, John Babich, and, of course, the Heidelberg group. Although the first steps were almost completely done in academia, we need industry for clinical translation. I am a strong supporter of strong industry relationships. However, much current debate focuses on how close such relationship should or can be. What is your perspective on this? 
Dr. Schaffert: Relationships between industry and academia are important to us. Although we have our own research efforts, we always balance this with licensing outside developments, often from academia. Successful academia-industry collaborations must be transparent. It is important to fully disclose all collaborations or ties. You need to follow the rules and respect each other.

Dr. Herrmann: What about the debate on insourcing vs. outsourcing? When all research is performed inside you have full control, but you also have a limited influx regarding ideas. For the field of theranostics, what do you think a company should really own and control, and what parts would you rather leave to outside sources?

Dr. Schaffert: The Novartis approach was always very balanced. We were conducting internal research, but also constantly exploring the market and looking for collaborations. That strategy also applies to AAA. You need a certain expertise inhouse-for AAA we have a dedicated research team mostly focused on the nuclear medicine part and dedicated to new targets. But there's a lot of great stuff outside in other companies and in academia that we also follow. Our key to success is to have a very balanced model. We would want to own any research post phase 2 , especially since we bring a lot of experience and know what the hurdles are, but we are very open about collaboration in earlier phases.

Dr. Herrmann: You just mentioned that AAA has an independent research team. Is this something completely new for Novartis?

Dr. Schaffert: Exactly. It is an innovative model. Novartis respects the growth mindset of AAA. Of course, you integrate certain interfaces. But quite unusual for Novartis we kept the research and production teams separate. Their skill and capability are mostly on the nuclear medicine side, and this is exactly where Novartis did not have experience. That's why we wanted to preserve that.
Dr. Herrmann: How can we accelerate clinical drug translation? Does it make sense to have certain proof-of-concept programs?

Dr. Schaffert: For theranostics, the solution could be diagnostic trials: to do them really quickly in phase 1 , because they already give us so much in terms of data, and then move on faster for therapeutic development. From the Novartis experience we learned that it is sometimes very difficult to select the right design for proof-of-concept trials. For me that is one of the big differences in nuclear medicine- that you learn so much from the diagnostic that you need less data on earlier phases. These are the data that are really important for regulatory bodies.

Dr. Herrmann: You just mentioned that you can actually go into humans comparatively earlier than for other therapies. Do you think there is still space for preclinical model systems for screening?

Dr. Schaffert: Very much so, because you need to first evaluate (especially when you are going to new targets) the amount of tumor expression, the specificity, and the amount of uptake. Moreover, preclinical models will also provide a lot of data that are needed for the regulatory package.

Dr. Herrmann: I am grateful for all the time you dedicated to this interview, and I think we covered a lot of ground. Is there anything else you want to tell us about theranostics?

Dr. Schaffert: No, I think you covered it very well. Theranostics is such a great new therapy option. But we have to make sure that theranostics gets established in the oncology curricula and in the oncologic treatment algorithms. This is something the industry, nuclear medicine, and oncologists must work on together. There is a rich opportunity now, but I think that it all depends on how you establish collaborations. That is very critical for me.

Dr. Herrmann: Thank you for these final comments, and thank you very much again for your time. 